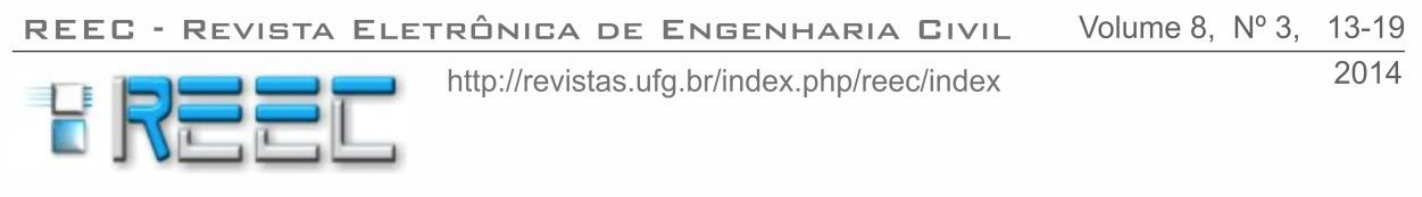

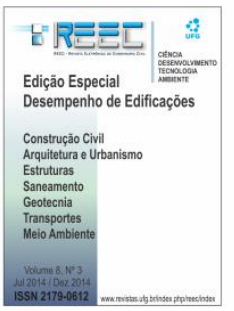

\title{
ESTUDO DE PARÂMETROS DA VENTILAÇÃO NATURAL PARA MAXIMIZAÇÃO DO CONFORTO TÉRMICO EM PAVILHÕES INDUSTRIAIS: VALIDAÇÃO EXPERIMENTAL EM TUNEL DE VENTO
}

\section{Study of parameters of natural ventilation to thermal comfort maximization in industrial pavilions: experimental analysis in wind tunnel}

\author{
Enaira Hoffmann de Oliveira ${ }^{1}$, Moacir Kripka ${ }^{2}$, Acir Mércio Loredo-Souza ${ }^{3}$
}

Recebido em 16 de fevereiro de 2014; recebido para revisão em 22 de março de 2014; aceito em 08 de abril de 2014; disponível on-line em 04 de junho de 2014.

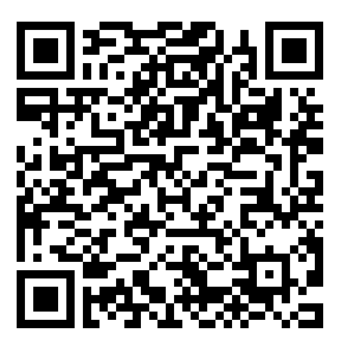

\section{PALAVRAS CHAVE:}

Pavilhões industriais;

Ventilação natural;

Conforto térmico;

Túnel de vento.

\section{KEYWORDS:}

Industrial buildings,

Natural ventilation,

Thermal comfort

RESUMO: Este trabalho apresenta resultados obtidos a partir de simulações com modelo reduzido em túnel de vento, com a finalidade de validar a aplicação de técnicas de otimização para a determinação de parâmetros de conforto ambiental nos ambientes industriais. Buscou-se maximizar o conforto térmico em pavilhões, com ou sem efeito do vento, tendo como variáveis de projeto as dimensões e as disposições das aberturas. Dentre outros resultados obtidos, verificou-se que, considerando o efeito do vento, há uma grande diminuição das áreas das aberturas calculadas, comparativamente aos casos em que a ventilação ocorre apenas por efeito chaminé.

ABSTRACT: This work presents some results obtained from the analysis of a reduced model in wind tunnel, aiming to validate the application of optimization techniques for the determination of parameters of environmental comfort in industrial buildings. In order to maximize the thermal comfort in pavilions, with or without considering the wind effect, the dimensions and arrangements of openings are taken as design variables. Among other results, it was found that, considering the effect of wind, a great reduction of the areas of the openings can be achieved, regarding to cases where only the ventilation chimney effect occurs.

\footnotetext{
* Contato com o autor:
}

${ }^{1}$ e-mail : arq.enaira@gmail.com

\section{( E. H. de Oliveira )}

Me, Programa de Pós-Graduação em Engenharia Civil e Ambiental - Universidade de Passo Fundo UPF

2e-mail : mkripka@upf.br

Prof. Dr., Programa de Pós-Graduação em Engenharia Civil e Ambiental - Universidade de Passo Fundo - UPF

3e-mail : 00009661@ufrgs.br

Prof. Dr., Programa de Pós-Graduação em Engenharia Civil - Universidade Federal do Rio Grande do Sul - UFRGS

\section{INTRODUÇÃO}

A preocupação com o aumento do consumo de energia para o condicionamento térmico das edificações e o bem estar de seus ocupantes incentiva a avaliação do desempenho ambiental dos edifícios. A ventilação natural propicia o condicionamento térmico dos ambientes e contribui para um bom desempenho ambiental dos edifícios. A movimentação natural do ar de forma adequada possibilita a renovação do ar no ambiente, além de diminuir a temperatura interna. 
Este tipo de estratégia de condicionamento térmico se faz por meio de aberturas (janelas, portas, lanternins, entre outros), que atuam como aberturas de entrada ou saída, e devem ser dimensionadas e estar posicionadas de modo a proporcionar um fluxo de ar adequado ao recinto. No projeto arquitetônico, o efeito da ventilação natural por ação do vento reside no posicionamento das aberturas em função das áreas de altas e baixas pressões na fachada, o que possibilita obter melhor aproveitamento desta estratégia de conforto (SHIMOMURA, FROTA e CELANI, 2010).

O conhecimento, em bases científicas, do fenômeno da ventilação natural, é relativamente pequeno comparado com o que atualmente existe sobre ventilação mecânica. Essa disparidade pode ser constatada pela bibliografia existente (NUNES, 2006). O presente artigo tem como objetivo validar a resultados obtidos a partir da aplicação de técnica de otimização na maximização da ventilação natural em pavilhões industriais, fornecendo subsídios para a determinação da disposição e do dimensionamento das aberturas visando um maior conforto térmico com redução no consumo de energia. Apesar de direcionada a pavilhões industriais, a metodologia proposta neste trabalho pode ser empregada para edificações com diferentes finalidades, como edifícios institucionais (escolas, unidades de saúde) e residências, sendo necessária a configuração das ferramentas utilizadas conforme a tipologia escolhida.

\section{DESENVOLVIMENTO METODOLÓGICO}

Com a finalidade de verificar o comportamento da vazão de ventilação por efeitos dos ventos de forma prática efetuou-se, além das análises numéricas, o ensaio de modelos reduzidos em túnel de vento. A vazão de ventilação nestes ensaios pode ser determinada por meio da medição direta da velocidade nas aberturas utilizando anemômetros de fio quente.

Para que a metodologia estudada fosse aplicada, utilizou-se como exemplo um modelo reduzido de pavilhão industrial semelhante ao utilizado por Silvani (2005). Para avaliar a influência da disposição das aberturas na edificação na vazão de ventilação promovida pelo vento, as áreas mínimas das aberturas das janelas foram dispostas em três configurações distintas.

Os ensaios foram realizados no Túnel de Vento Prof. Joaquim Blessmann (Figura 1) da Universidade Federal do Rio Grande do Sul. Trata-se de um túnel de vento de retorno fechado, projetado especificamente para ensaios estáticos e dinâmicos de modelos de construções civis em operação desde 1977.

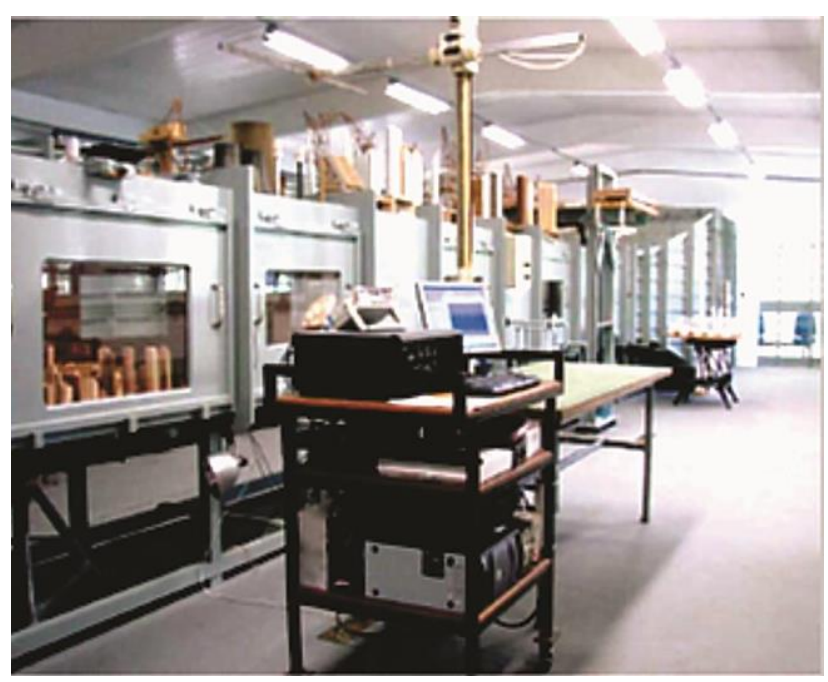

FIGURA 1: Túnel de vento Prof. Joaquim Blessmann Universidade Federal do Rio Grande do Sul.

A utilização do anemômetro de fio quente permite encontrar valores para as velocidades pontuais. Com isso, é possível explorar as velocidades locais de vários pontos de uma abertura, segundo uma metodologia apropriada, e deduzir a vazão, mesmo considerando que o escoamento do ar difere quando comparado no centro e nas bordas das aberturas. Conhecendo-se a velocidade no centro ou deduzindo-se a velocidade média a partir da média das velocidades ao longo de uma abertura, pode-se determinar o 
perfil das velocidades em função do numero de Reynolds.

A média das velocidades obtidas nos anemômetros de fio quente é calculada a partir do levantamento das velocidades tomadas em intervalos regulares ao longo de cada abertura. Conforme a equação de Bernoulli, a velocidade média do ar que passa por uma abertura é dada por meio da Equação 01:

$$
V_{\text {média }}=\sqrt{\frac{2 . \Delta p}{\rho_{a r}}}
$$

Onde:

$\Delta p=$ diferencial de pressão através do orifício $(\mathrm{Pa})$; $\rho_{\text {ar }}=$ massa especifica do ar que passa pelo orifício $\left(\mathrm{Kg} / \mathrm{m}^{3}\right)$;

$\mathrm{V}_{\text {média }}=$ velocidade média do $\operatorname{ar}(\mathrm{m} / \mathrm{s})$.

A vazão teórica $Q_{\text {teórica }}$ é expressa pela Equação 02:

$$
Q_{\text {teórica }}=A . V_{\text {média }}
$$

Onde:

$A=$ área da abertura $\left(\mathrm{m}^{2}\right)$;

$\mathrm{V}_{\text {média }}=$ velocidade média do $\operatorname{ar}(\mathrm{m} / \mathrm{s})$.

Contudo, para se obter a vazão real, devese considerar um coeficiente de vazão, $K_{v}$. Este coeficiente descreve a relação entre o diferencial de pressão através da abertura e a vazão de ar que passa através dela. Em outras palavras, $K_{v}$ é uma medida de eficiência das aberturas a passagem de ar, conforme Equação 03:

$$
K_{v}=\frac{Q_{\text {real }}}{Q_{\text {teórico }}}
$$

Onde:

$Q_{\text {real }}=$ vazão real na abertura $\left(\mathrm{m}^{3} / \mathrm{s}\right)$;

$Q_{\text {teórico }}=$ vazão teórica na abertura $\left(\mathrm{m}^{3} / \mathrm{s}\right)$.

O modelo do pavilhão industrial utilizado nos ensaios, com escala 1:200, foi confeccionado em material acrílico de $2 \mathrm{~mm}$ de espessura. 0 exaustor natural de cumeeira foi executado em chapa metálica, com detalhes mais precisos da abertura. Todas as aberturas existentes no modelo foram reproduzidas com vãos livres para a passagem do escoamento, com exceção dos portões que foram considerados fechados (Figura 2).

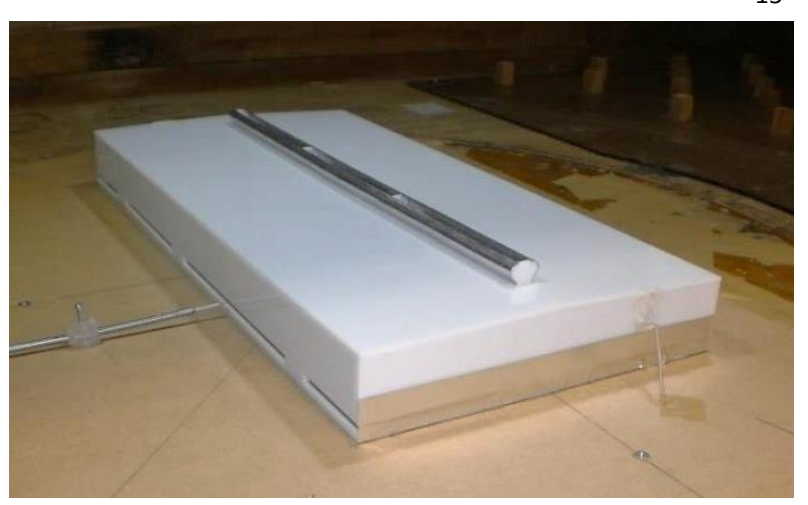

FIGURA 2: Modelo do pavilhão industrial com as aberturas livres para passagem do escoamento.

As áreas das aberturas otimizadas dimensionadas no modelo do pavilhão foram encontradas utilizando a formulação proposta por Clezar e Nogueira (1999) descrita em planilha Excel desenvolvida pelos autores.

Com isso, posicionou-se uma sonda em cada uma das três principais aberturas do modelo, sendo que as medições foram realizadas de forma simultânea para as três sondas (Figura 3). O fio aquecido foi posicionado perpendicularmente ao escoamento de cada abertura, permitindo medir as velocidades médias pontuais de entrada ou saída de ar (Figura 4).

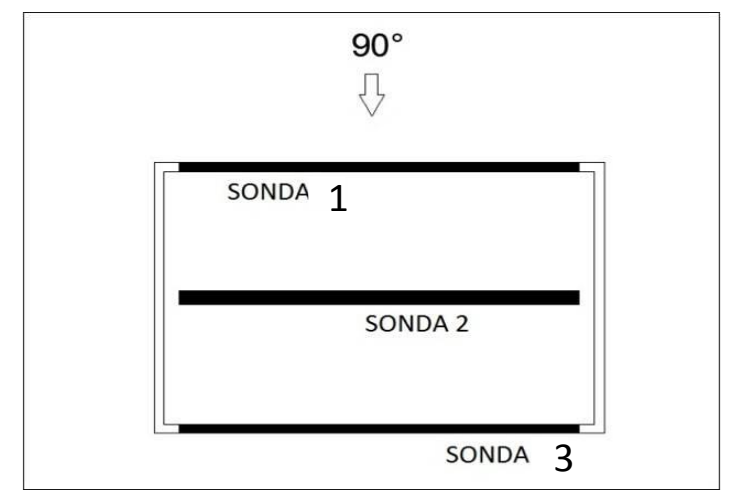

FIGURA 3: Planta baixa do pavilhão - localização das sondas.

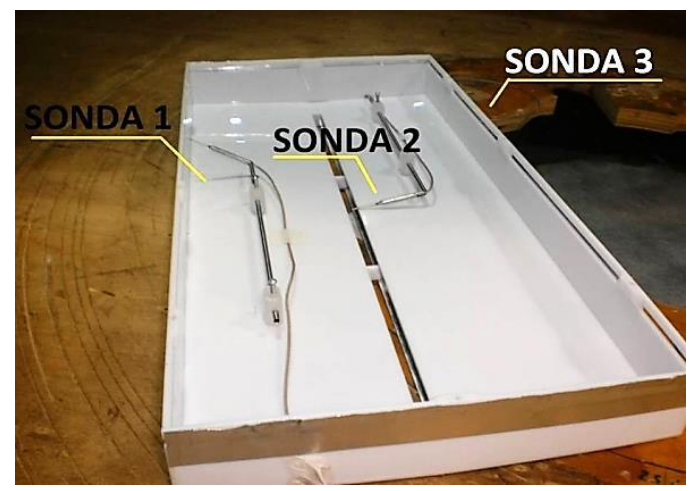

FIGURA 4: Modelo do pavilhão- localização das sondas. 
Com o posicionamento das sondas, foram ensaiadas as três configurações de fechamento de aberturas. Para cada uma das três configurações foram simulados dois tipos de vento, com perfil de velocidades médias horárias, $p=0,11$ e $p=0,23$, correspondentes respectivamente às categorias de rugosidade superficial (tipo de terreno) I e III/IV da Norma NBR-6123/88, sendo que para cada tipo de vento simulado foram ensaiadas duas velocidades médias de escoamento no túnel, uma velocidade com as aletas do túnel totalmente abertas (V1) e outra com as aletas do túnel parcialmente abertas (V2). Os ensaios foram realizados com o modelo fixado a mesa giratória M-II, na câmara de ensaios, para o ângulo de $90^{\circ}$, obtendo com isso as velocidades medias pontuais no centro das aberturas onde foram posicionadas as sondas (anemômetro de fio aquecido).

Para a determinação da vazão de ventilação nas aberturas a barlavento do pavilhão utilizando os resultados obtidos dos ensaios foram consideradas ainda as seguintes hipóteses:

- A vazão de ventilação nas aberturas foi determinada considerando como coeficiente de vazão, $\mathrm{K}_{\mathrm{v}}=0,613$ para vento $p=0,11$ e $\mathrm{K}_{\mathrm{v}}=0,410$ para vento $p=0,23$;

- As velocidades médias adimensionais nas aberturas do modelo em escala reduzida foram consideradas iguais as médias das velocidades adimensionais pontuais obtidas nas medições realizadas com os anemômetros;

- As velocidades médias nas aberturas do pavilhão industrial utilizado como modelo foram determinadas através da multiplicação das velocidades médias adimensionais das aberturas do modelo em escala reduzida pela velocidade média do vento para o protótipo;

- A velocidade do vento foi considerada igual a $2,0 \mathrm{~m} / \mathrm{s}$ para uma velocidade do vento correspondente a $50 \%$ da velocidade média sazonal para o protótipo. Essa velocidade média igual a $4 \mathrm{~m} / \mathrm{s}$ foi obtida dos registros da Embrapa Trigo - Passo Fundo, correspondentes aos meses de dezembro, janeiro, fevereiro e março, durante o período de 1977 a 1994 (EMBRAPA, 2004).

\section{RESULTADOS OBTIDOS}

Nas Tabelas 1 a 3 são apresentadas as vazões de ar nas aberturas situadas nas fachadas a barlavento do pavilhão, obtidas através das velocidades adimensionais determinadas nos ensaios. As aberturas do pavilhão estão posicionadas conforme as Figuras 5 a 7.

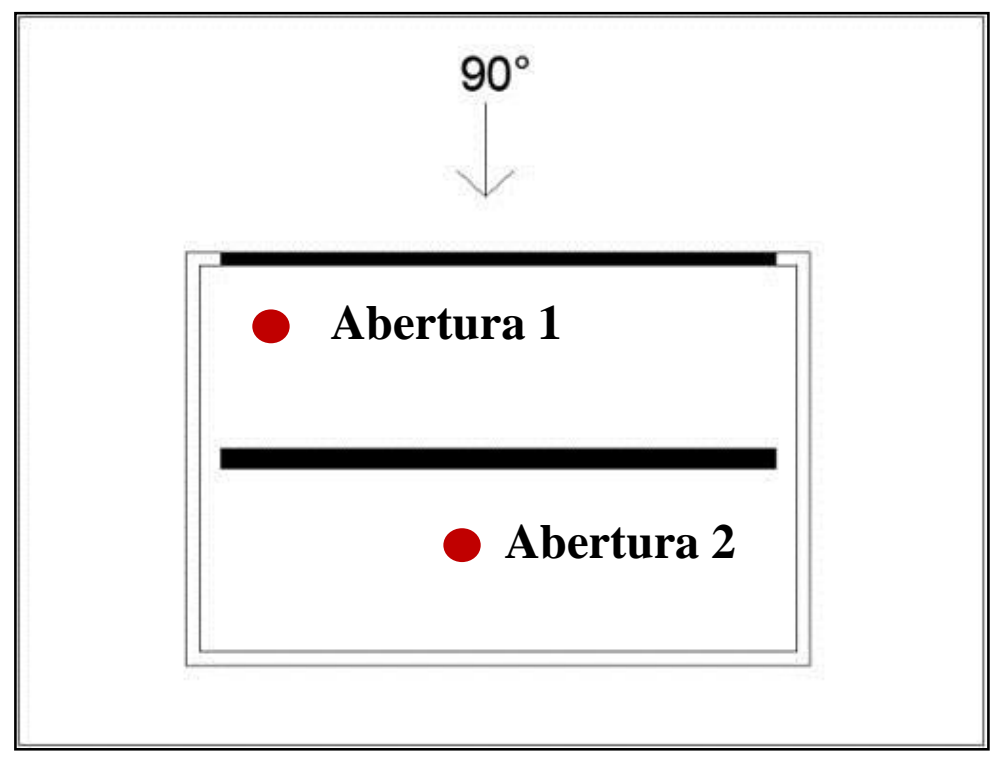

FIGURA 5: Posição das aberturas no pavilhão 1 - planta baixa. 
TABELA 1: Vazões de ar nas aberturas do pavilhão com configuração 1 para vento simulado $p=0,11$ e $p=0,23$ e velocidade média do vento igual a $2 \mathrm{~m} / \mathrm{s}$.

\begin{tabular}{|c|c|c|c|c|c|}
\hline \multirow{2}{*}{ Vento } & $\begin{array}{c}\text { Posição da } \\
\text { abertura no } \\
\text { pavilhão }\end{array}$ & $\mathrm{Kv}$ & $\begin{array}{c}\text { Área da } \\
\text { abertura } \\
\mathrm{A}\left(\mathrm{m}^{2}\right)\end{array}$ & $\begin{array}{c}\text { Velocidade média na } \\
\text { abertura } \\
\mathrm{V}(\mathrm{m} / \mathrm{s})\end{array}$ & $\begin{array}{c}\text { Vazão de ar } \\
\mathrm{Q}\left(\mathrm{m}^{3} / \mathrm{s}\right)\end{array}$ \\
\hline \multirow{2}{*}{$p=0,11$} & 1 & 0,613 & 70,19 & 6,562 & 282,33 \\
\cline { 2 - 6 } & 2 & 0,613 & 71,79 & 0,485 & 21,34 \\
\hline \multirow{2}{*}{$p=0,23$} & 1 & 0,410 & 70,19 & 5,667 & 163,08 \\
\cline { 2 - 6 } & 2 & 0,410 & 71,79 & 0,393 & 11,56 \\
\hline
\end{tabular}

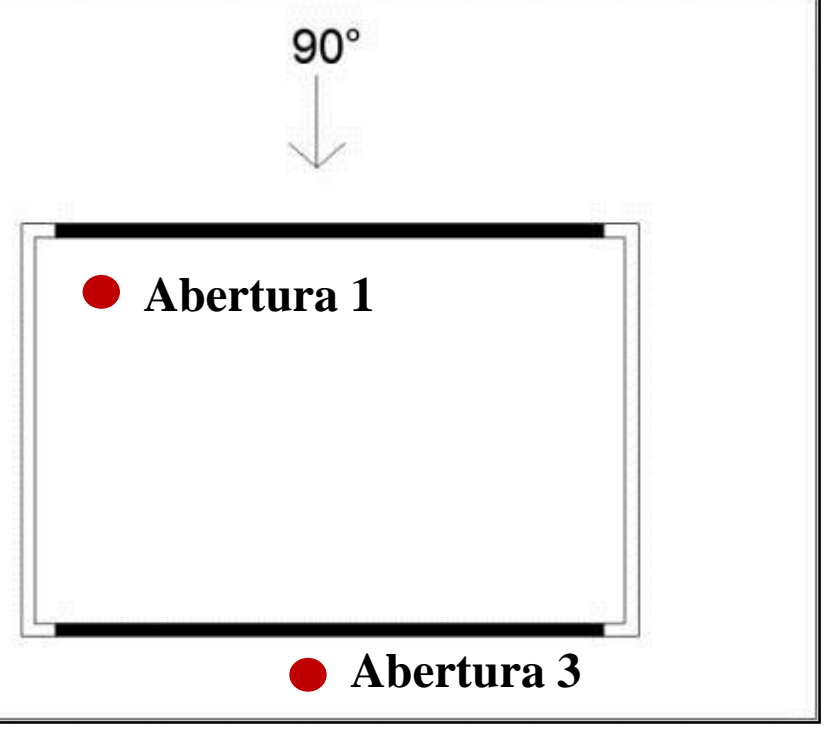

FIGURA 6: Posição das aberturas no pavilhão 2 - planta baixa.

TABELA 2: Vazões de ar nas aberturas do pavilhão com configuração 2 para vento simulado $p=0,11$ e $p=0,23$ e velocidade média do vento igual a $2 \mathrm{~m} / \mathrm{s}$.

\begin{tabular}{|c|c|c|c|c|c|}
\hline \multirow{2}{*}{ Vento } & $\begin{array}{c}\text { Posição da } \\
\text { abertura no } \\
\text { pavilhão }\end{array}$ & $\mathrm{Kv}$ & $\begin{array}{c}\text { Área da } \\
\text { abertura } \\
\mathrm{A}\left(\mathrm{m}^{2}\right)\end{array}$ & $\begin{array}{c}\text { Velocidade } \\
\text { média na } \\
\text { abertura } \\
\mathrm{V}(\mathrm{m} / \mathrm{s})\end{array}$ & $\begin{array}{c}\text { Vazão de } a r \\
\mathrm{Q}\left(\mathrm{m}^{3} / \mathrm{s}\right)\end{array}$ \\
\hline $\mathrm{p}=0,11$ & 1 & 0,613 & 126,23 & 6,033 & 466,82 \\
\cline { 2 - 6 } & 3 & 0,613 & 126,23 & 0 & 0 \\
\hline $\mathrm{p}=0,23$ & 1 & 0,410 & 126,23 & 5,206 & 269,43 \\
\cline { 2 - 7 } & 3 & 0,410 & 126,23 & 0 & 0 \\
\hline
\end{tabular}




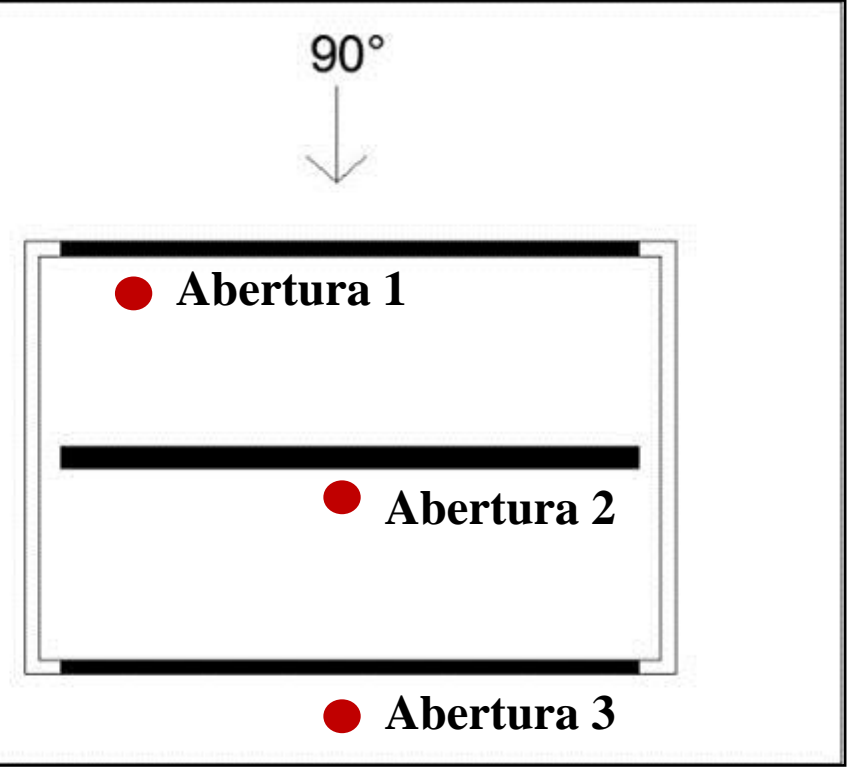

FIGURA 7: Posição das aberturas no pavilhão 2 - planta baixa.

\begin{tabular}{|c|c|c|c|c|c|}
\hline Vento & $\begin{array}{l}\text { Posição da } \\
\text { abertura no } \\
\text { pavilhão }\end{array}$ & $\mathrm{Kv}$ & $\begin{array}{l}\text { Área da } \\
\text { abertura } \\
\mathrm{A}\left(\mathrm{m}^{2}\right)\end{array}$ & $\begin{array}{c}\text { Velocidade média } \\
\text { na abertura } \\
\text { V (m/s) }\end{array}$ & $\begin{array}{c}\text { Vazão de ar } \\
\mathrm{Q}\left(\mathrm{m}^{3} / \mathrm{s}\right)\end{array}$ \\
\hline \multirow{3}{*}{$p=0,11$} & 1 & 0,613 & 90 & 7,963 & 439,31 \\
\hline & 2 & 0,613 & 67,65 & 1,877 & 77,86 \\
\hline & 3 & 0,613 & 90 & 1,733 & 95,60 \\
\hline \multirow{3}{*}{$p=0,23$} & 1 & 0,410 & 90 & 6,673 & 246,23 \\
\hline & 2 & 0,410 & 67,65 & 1,086 & 30,13 \\
\hline & 3 & 0,410 & 90 & 1,254 & 46,27 \\
\hline
\end{tabular}

Com o fechamento de algumas aberturas do pavilhão nos ensaios no túnel de vento, ocorreram alterações nas vazões de ar. Percebeu-se que, à medida que as aberturas vão sendo fechadas, a vazão de ventilação diminui. Os exaustores de cumeeira (lanternins) são normalmente associados à ventilação promovida pela diferença de temperatura (efeito chaminé) auxiliando a ventilação promovida pelo efeito do vento. Observou-se, para a configuração 2, um expressiva diminuição da vazão ao considerar o exaustor de cumeeira do pavilhão fechado.

Com a análise comparativa, observou-se que as vazões de ar, parâmetro inicial encontrado através do cálculo do número de trocas de ar e volume do pavilhão, foram menores que as encontradas através dos ensaios em túnel de vento, destacando-se que, nos ensaios em métodos reduzidos, é possível chegar mais próximo das características reais do ambiente onde a edificação 
está inserida.

O ensaio do pavilhão industrial no túnel de vento resultou em vazões diferentes de entrada e de saída, devido às variações de magnitude da velocidade média do vento no túnel. Destaca-se que dependendo da posição da abertura do modelo, as velocidades adimensionais não são proporcionais à magnitude da velocidade media de escoamento no túnel. As velocidades de entrada e saída de ar nas aberturas dependem do tipo de vento simulado, da magnitude da velocidade do vento, da posição das aberturas e da configuração de fechamento das aberturas da edificação em questão.

\section{CONCLUSÕES}

Especificamente com relação ao estudo desenvolvido com modelo reduzido em túnel de vento, os resultados obtidos permitiram concluir que:

- A medição direta de velocidades através de anemômetro de fio quente permite que se obtenham vazões de ventilação sem iterações, com resultados próximos aos modelos teóricos estudados que se baseiam na diferença de pressões internas e externas;

- As velocidades de entrada ou saída de ar nas aberturas dependem do perfil do vento, magnitude da velocidade do vento, da posição e dimensionamento das aberturas em pavilhões;

- As aberturas localizadas nas cumeeiras são fatores importantes na ventilação promovida pela diferença de temperatura (efeito chaminé). Possuem fundamental importância para a ventilação promovida pelo vento porque permitem a diminuição dos vãos nas aberturas.

\section{AGRADECIMENTOS}

Os autores agradecem a Coordenação de Aperfeiçoamento de Pessoal de Nível Superior CAPES pelo apoio financeiro para a realização desta pesquisa.

\section{REFERÊNCIAS BIBLIOGRÁFICAS}

ASSOCIAÇÃO BRASILEIRA DE NORMAS TÉCNICAS. NBR 6123: Forças devidas ao vento em edificações. São Paulo, 1988.

CLEZAR, C. A.; NOGUEIRA, C. R. Ventilação industrial. Florianópolis: UFSC, 1999.

EMBRAPA, Embrapa Trigo de Passo Fundo. Disponível em: < http://www.cnpt.embrapa.br/>. Último acesso em 14 de jul. 2011.

NUNES, D. A. Estudo da ventilação natural por efeito do vento em pavilhões industriais utilizando modelos reduzidos. Dissertação (Mestrado em Engenharia Civil) Universidade Federal do Rio Grande do Sul, Porto Alegre, 2006.

SHIMOMURA, A.P.; FROTA, A. B.; CELANI, M. G. $C$. Modelos físicos na análise de ventilação urbana: o uso do túnel de Vento. Fórum Patrimônio: Ambiente Construído e Patrimônio Sustentável, v. 4, n.1, p. 1-22, 2010.

SILVANI, M. Subsídios para o projeto da ventilação natural em pavilhões industriais. Dissertação (Mestrado em Engenharia) - Faculdade de Engenharia e Arquitetura, Universidade de Passo Fundo, Passo Fundo, 2005. 